\title{
A Novel Dual-Mode Dual-Band Bandpass Filter Based on a Single Ring Resonator
}

\author{
Youngje Sung*
}

\begin{abstract}
In this paper, a dual-mode bandpass filter that operates in two different bands is proposed. A circular patch with a small circular hole is set as the basic resonator, and, as the circular hole moves, the linewidth of the ring resonator becomes non-uniform. Consequently, the ring resonator is structurally asymmetric and exhibits a dual-mode characteristic. A gap is formed in the middle of the resonator, a pair of inductors and capacitors is applied to the gap, and the proposed dual-mode structure operates as a dual-band filter. The first resonant frequency $f_{1}$ is determined by the inductor and capacitor values, and the second resonant frequency $f_{2}$ is determined by the capacitor values. In addition, the frequency ratio $\left(f_{2} / f_{1}\right)$ can be adjusted by an appropriate combination of inductor and capacitor values.
\end{abstract}

Key Words: Dual-Band Reconfigurable Antenna, Polarization-Reconfigurable Antenna, Switchable Orthogonal Linear Polarizations.

\section{INTRODUCTION}

Recently, developments in wireless communication technology have seen a diversification of the communication services provided to single mobile terminals. Accordingly, dual- and multi-band microwave components are preferred for such communication systems [1], and many dual-band dual-mode filters have been studied [2-5]. To realize dual-band dual-mode characteristics, a new method of cascading two resonators has been presented [2], as well as, elsewhere, a substrate-integrated waveguide structure [3], a stepped-impedance stub resonator [4], and a stepped-impedance resonator and embedded coupled-line [5]. However, none of these structures can adjust the two resonant frequencies independently.

To address this, a dual-band dual-mode bandpass filter (BPF) based on a multi-layer structure has previously been designed [6, 7]. Dual-band operation can thereby be obtained by separately implementing a resonator on each of the two substrates; this structure is advantageous in that each operating band can be controlled independently. However, the multi-layer structure faces other challenges in terms of adjusting the alignment between the resonators on the different layers.

A dual-band dual-mode BPF implemented on a single layer is preferred because it is simpler in structure and easy to fabricate. Generally, to obtain a dual-band characteristic on a singlelayer, an electrical path for each operating band must be implemented separately [8-10].

In this study, a novel dual-band dual-mode BPF based on a single microstrip ring resonator was designed. Unlike conventional dual-mode filters that use ring resonators, this filter can easily obtain a dual-band characteristic by using a non-uniform linewidth ring without an additional perturbation structure. In the proposed filter, the dual-band characteristic is obtained by simultaneously applying an inductor and a capacitor to the gap, and by combining various inductor and capacitor values, the frequency ratio of the filter can be adjusted from 1.31 to 1.83 .

\footnotetext{
Manuscript received September 7, 2019 ; Revised October 29, 2019 ; Accepted December 20, 2019. (ID No. 20190907-075J)

Department of Electronic Engineering, Kyonggi University, Suwon, Korea.

"Corresponding Author: Youngje Sung (e-mail: yjsung@kgu.ac.kr)
}

This is an Open-Access article distributed under the terms of the Creative Commons Attribution Non-Commercial License (http://creativecommons.org/licenses/by-nc/4.0) which permits unrestricted non-commercial use, distribution, and reproduction in any medium, provided the original work is properly cited.

(c) Copyright The Korean Institute of Electromagnetic Engineering and Science. All Rights Reserved. 


\section{GEOMETRY}

Fig. 1 shows the proposed dual-band dual-mode BPF using a single ring resonator with a non-uniform linewidth. The proposed filter structure is implemented using a substrate with a dielectric constant of 10.2 and a thickness of $50 \mathrm{mil}$, and size 1005 Murata chip inductors and capacitors are used.

If the linewidth of the ring resonator is constant, the structure operates as a single-mode filter. Typically, to obtain a dualmode characteristic using this structure, a cut or stub is applied to change the line impedance at $45^{\circ}$ and $135^{\circ}$ with respect to the input and output stages. In the proposed structure, the linewidth of the ring resonator is unevenly formed, and the line impedances at $45^{\circ}$ and $135^{\circ}$ are automatically changed with respect to the input and output stages, thereby realizing the dualmode characteristic.

The feed is implemented by a circular arc, and the linewidth of the feed is set to $w$. The gap between the feed line and the resonator is set to $g$, and the angle is set to $\alpha$. In this study, the parameters of the feed structure are optimized using an HFSS simulator.

Fig. 2 compares the frequency response of the proposed filter

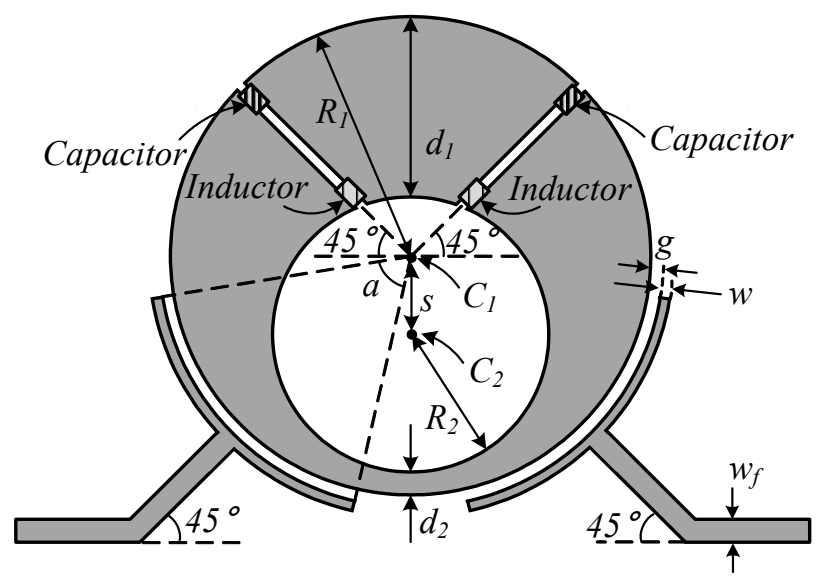

Fig. 1. The proposed dual-band dual-mode filter.

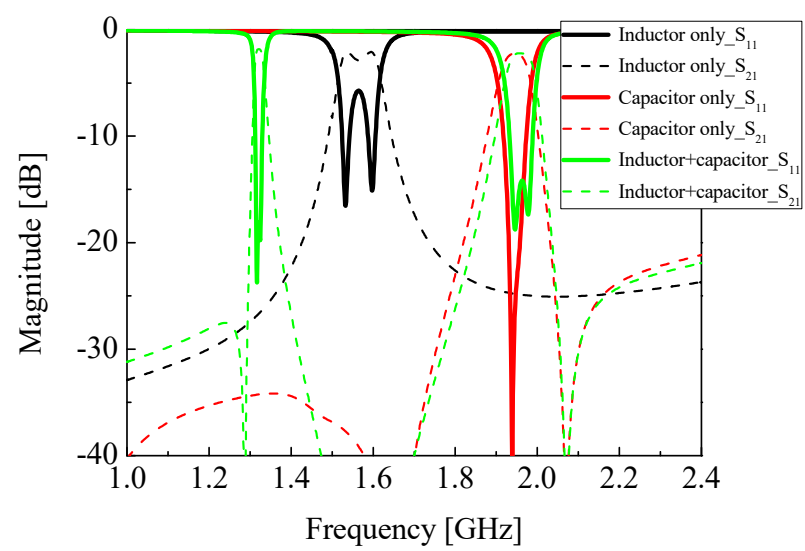

Fig. 2 Simulated frequency responses with different dual-mode filters. in structures where only the inductor ("Inductor only") or the capacitor ("Capacitor only") is applied.

As shown, the resonant frequency of the filter is formed at $1.55 \mathrm{GHz}$ in the case of the inductor-only filter. As the capacitor is added, the resonant frequency of the filter is lowered to the $1.3 \mathrm{GHz}$ band which is the first resonant frequency of the proposed filter. The first resonant frequency can therefore be adjusted by changing the capacitor or inductor value. Meanwhile, the frequency response near $1.95 \mathrm{GHz}$, the second resonant frequency, is similar to that of the capacitor-only structure. This demonstrates that the second resonant frequency of the proposed structure is determined by the capacitor value.

\section{PARAMETER STUDY}

Fig. 3 shows the simulated frequency response with different inductors $L$. At this time, $C$ is fixed at $2.8 \mathrm{pF}$. The other $\mathrm{pa}^{-}$ rameters are as follows: $R_{1}=12 \mathrm{~mm}, R_{2}=8 \mathrm{~mm}, s=2 \mathrm{~mm}, d_{1}$ $=3 \mathrm{~mm}, d_{2}=1 \mathrm{~mm}, g=w=0.2 \mathrm{~mm}$, and $\alpha=60^{\circ}$. Although not shown, resonance does not appear in the low frequency band when inductors are absent, only appearing at $1.95 \mathrm{GHz}$. When the inductor value is $0.3 \mathrm{nH}$, a weak resonance is formed at $1.37 \mathrm{GHz}$. As the inductor value becomes larger, resonant frequency in the low frequency band decreases, and the two resonance modes are gradually split. At the same time, there is almost no change in the high frequency band. To obtain a proper dual-mode characteristic in the low-frequency band, $L$ is therefore selected at $0.5 \mathrm{nH}$.

Fig. 4 shows the simulated frequency response with different capacitors $C$. At this time, the $0.5 \mathrm{nH}$ inductor $L$ is applied, and the other parameters are the same as above. As the capacitor value increases, the frequency response of both bands tends to decrease simultaneously. As $C$ increases, the two resonances split in the low frequency band but combine in the high frequency band.

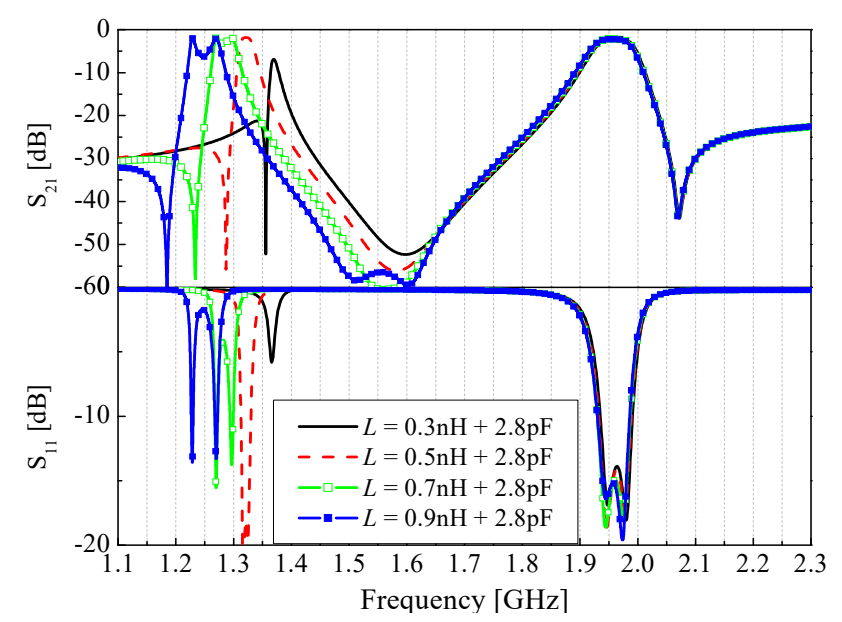

Fig. 3. Simulated frequency responses with different inductors $L$. 


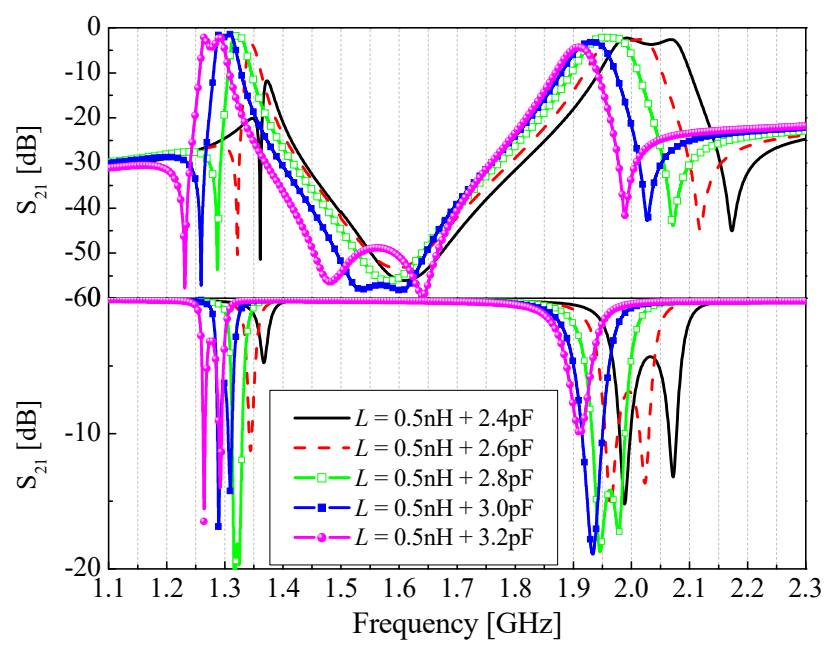

Fig. 4. Simulated frequency responses with different capacitors $C$.

Fig. 5 shows the frequency response of the proposed filter as the distance $s$ changes. As $s$ increases, the low frequency band decreases downward, but the high frequency band barely changes. Further, as $s$ increases, the two resonances tend to combine in the two bands. The process of designing the proposed dualband dual-mode BPF can therefore be presented as follows:

(1) Set $R_{1}, R_{2}$, and $s$;

(2) Set $C$ to exhibit high frequency dual-mode characteristic;

(3) Add $L$ to the gap to implement dual-mode characteristic in the low frequency band. The capacitor value $C$ in the previous step will requires slight tuning;

(4) Optimum results can be obtained by tuning the feed.

According to this design strategy, various $L$ and $C$ combinations are applied to the original structure (Fig. 1), and the results are presented in Fig. 6.

The parameter $s$ is adjusted to obtain the appropriate filter characteristic. As $L$ and $s$ increase, the resonant frequency in the low and high bands decrease and increase, respectively. Meanwhile, as $C$ increases, the resonant frequency in the low and

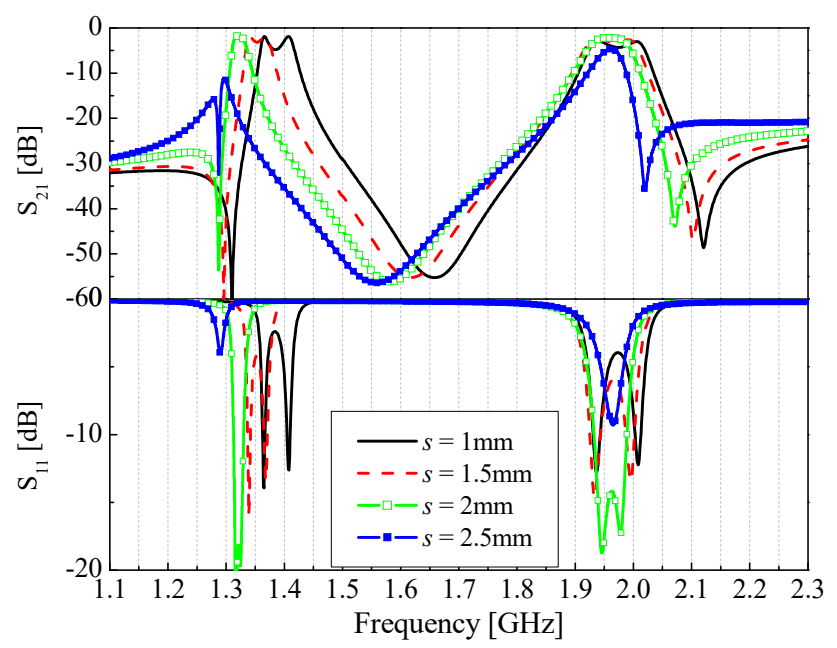

Fig. 5. Simulated frequency responses with different distances $s$.

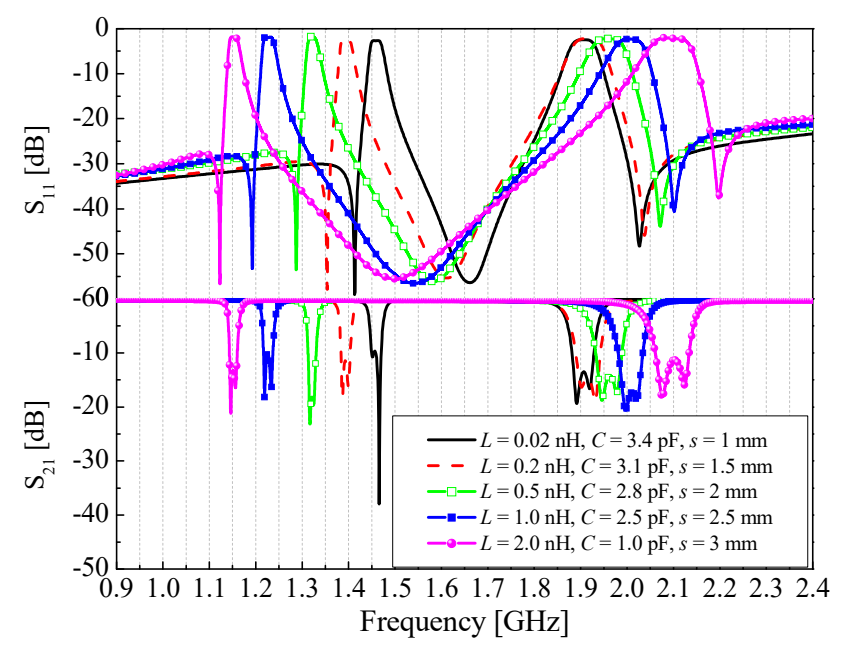

Fig. 6. Simulated frequency responses with various combinations of $L$ and $C$.

high bands increases and decreases, respectively. As a consequence, the frequency ratio between the two operating bands changes.

\section{SIMULATED AND MEASURED RESULTS}

Fig. 7 shows the simulated and measured frequency responses of a fabricated filter. The parameters here are $R_{1}=12 \mathrm{~mm}, R_{2}$ $=8 \mathrm{~mm}, s=2 \mathrm{~mm}, d_{1}=3 \mathrm{~mm}, d_{2}=1 \mathrm{~mm}, g=w=0.2 \mathrm{~mm}, \alpha$ $=60^{\circ}, L=0.5 \mathrm{nH}$, and $C=2.8 \mathrm{pF}$.

The measured passbands are centered at $1.31 \mathrm{GHz}$ and 1.97 $\mathrm{GHz}$ with a $3-\mathrm{dB}$ fractional bandwidth of $1.1 \%$ and $3.4 \%$, respectively. The simulated minimum insertion losses of each band are $-1.71 \mathrm{~dB}$ and $-2.1 \mathrm{~dB}$ while the measured minimum insertion losses are $-1.86 \mathrm{~dB}$ and $-2.32 \mathrm{~dB}$. Three transmission zeros with frequency locations of $1.23 \mathrm{GHz}, 1.53 \mathrm{GHz}$, and $2.01 \mathrm{GHz}$ can be clearly observed, providing sharp band-toband rejection.

The slight discrepancy in these results is attributed to fabrication tolerance as well as the SMA connectors which were not

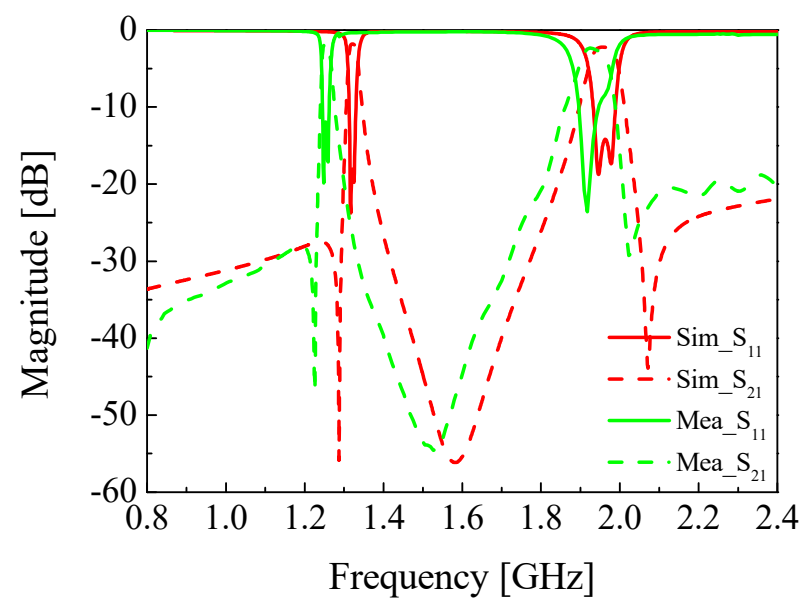

Fig. 7. Simulated and measured results of the proposed filter. 


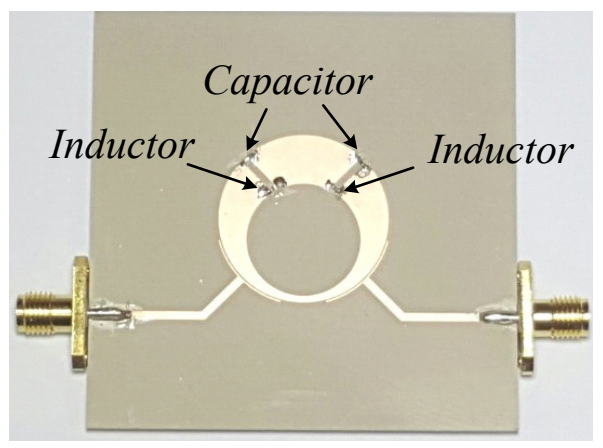

Fig. 8. The fabricated dual-band dual-mode filter.

considered in the simulation. The existence of insertion loss is mainly due to conductor and dielectric circuit losses and could be improved by more careful fabrication and better measurement technology. Fig. 8 presents the fabricated dual-band dualmode filter.

\section{CONCLUSION}

We have proposed a novel dual-band dual-mode BPF using a ring resonator with non-uniform linewidth. The operating principles of the proposed filter are presented above, and the nonuniform linewidth of the ring is created by off-setting the patch and hole centers. The proposed filter is characteristically dualmode; inductors and capacitors are applied to the gap, causing the filter to operate in two different bands. In addition, the frequency ratio of the proposed filter can be adjusted from 1.31 to 1.83 through various combinations of inductor and capacitor values.

This work was supported by the National Research Foundation of Korea funded by the Korea government (No. 2017R1A2B4010393).

\section{REFERENCES}

[1] H. S. Im and S. W. Yun, "Design of a dual-band bandpass er using an open-loop resonator," Journal of Electromagnetic Engineering and Science, vol. 17, no. 4, pp. 197-201, 2017.

[2] S. Fu, B. Wu, J. Chen, S. J. Sun, and C. H. Liang, "Novel second-order dual-mode dual-band filters using capacitance loaded square loop resonator," IEEE Transactions on Microwave Theory and Techniques, vol. 60, no. 3, pp. 477-483, 2012.

[3] K. Zhou, C. X. Zhou, and W. Wu, "Resonance characteristics of substrate-integrated rectangular cavity and their applications to dual-band and wide-stopband bandpass filters design," IEEE Transactions on Microwave Theory and Techniques, vol. 65, no. 5, pp. 1511-1524, 2017.

[4] S. Yang, L. Lin, J. Chen, K. Deng, and C. H. Liang, "Design of compact dual-band bandpass filter using dual-mode stepped-impedance stub resonators," Electronics Letters, vol. 50, no. 8, pp. 611-613, 2014.

[5] H. Zhu and A. M. Abbosh, "Single-and dual-band bandpass filters using coupled stepped-impedance resonators with embedded coupled-lines," IEEE Microwave and Wireless Components Letters, vol. 26, no. 9, pp. 675-677, 2016.

[6] E. E. Djoumessi and K. Wu, "Multilayer dual-mode dualbandpass filter," IEEE Microwave and Wireless Components Letters, vol. 19, no. 1, pp. 21-23, 2009.

[7] J. X. Chen, C. Shao, J. Shi, and Z. H. Bao, "Multilayer independently controllable dual-band bandpass filter using dualmode slotted-patch resonator," Electronics Letters, vol. 49, no. 9, pp. 605-607, 2013.

[8] J. Wang, L. Ge, K. Wang, and W. Wu, "Compact microstrip dual-mode dual-band bandpass filter with wide stopband," Electronics Letters, vol. 47, no. 4, pp. 263-265, 2011.

[9] J. Lee and Y. Lim, "Compact dual-band bandpass filter with good frequency selectivity," Electronics Letters, vol. 47, no. 25, pp. 1376-1377, 2011.

[10] X. Huang, L. Zhu, Q. Feng, Q.Xiang, and D. Jia, "Tunable bandpass filter with independently controllable dual passbands," IEEE Transactions on Microwave Theory and Techniques, vol. 61, no. 9, pp. 3200-3208, 2013. 
Youngje Sung

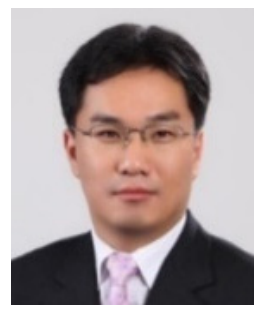

received B.S., M.S., and Ph.D. degrees from Korea University, Seoul in 2001, 2002, and 2005, respectively. From 2005 to 2008, he was a Senior Engineer with the Antenna R\&D Laboratory, Mobile Phone Division at Samsung Electronics. In 2008, he joined the Department of Electronic Engineering at Kyonggi University, Suwon, Korea where he is currently a professor. His research interests include reconfigurable antennas, cellphone antennas, wideband slot antennas, multifunction devices, compact circular polarized antennas, and compact dualmode filters. Prof. Sung currently serves as a reviewer for IEEE Transactions on Microwave Theory and Techniques, IEEE Transactions on Antennas and Propagation, IEEE Microwave and Wireless Components Letters, IEEE Antennas and Wireless Propagation Letters, Progress in Electromagnetic Research, IET Electronics Letters, and IET Microwaves, and Antennas and Propagation. 